\title{
Associação entre sintomatologia depressiva e óbito hospitalar em idosos
}

\author{
Association between depressive symptomatology \\ and hospital death in elderly \\ Rilva Lopes de Sousa-Muñoz', Ezemir Dantas Fernandes Junior², Divany de Brito Nascimento², \\ Bruno Braz Garciaª , Iramirton Figuêredo Moreira²
}

\section{RESUMO}

Objetivo: Avaliar se a presença de sintomas depressivos associa-se a uma evolução hospitalar desfavorável (aumento da permanência e mortalidade hospitalar), independente da capacidade funcional. Método: Coorte prospectivo em pacientes idosos internados nas enfermarias de Clínica Médica do Hospital Universitário Lauro Wanderley (HULW), avaliados em dois momentos (segundo dia de internação e na alta). Os instrumentos utilizados foram a Escala de Depressão Geriátrica, versão breve (EDG-15) e Índice de Barthel para Incapacidade em Atividades da Vida Diária (IBAIVD). Resultados: A idade dos 100 pacientes variou entre 60 e 96 anos (69,47 $\pm 7,45$ ). A mortalidade hospitalar relacionou-se com os escores da EDG-15 da internação ( $p=$ $0,001)$. Observou-se correlação entre os escores da EDG-15 e IBAIVD no início $(p=0,008)$ e final da internação $(p=0,01)$, verificando-se correlações lineares inversas $(p=-0,30)$, porém de magnitudes fracas ( $p=-0,30$ e $p=-0,28$, respectivamente). Conclusão: Sintomatologia depressiva

\section{Palavras-chave}

Depressão, idosos, mortalidade.

\section{Keywords}

Depression, elderly, mortality. associou-se à maior mortalidade, independente da capacidade funcional. Ressalta-se a importância da avaliação da sintomatologia depressiva nos idosos internados. Novos estudos e análises prospectivas poderão oferecer mais indicadores sobre esse problema de pesquisa.

\section{ABSTRACT}

Objective: Evaluate whether the depressive symptoms are associated to an unfavorable hospital course (increased hospital stay and mortality), independent of the functional capacity. Method: Cohort-longitudinal and the inpatients in Medical Clinical on University Hospital Lauro Wanderley were submitted to interview in $2^{\text {nd }}$ and in the day of hospital discharge. The GDS-15 (Geriatric Depression Scale) was utilizated to assess the depressive symptoms and the Barthel Indice to assess the disability in Activities of Daily Living. Results: The age of hundred patients were between 60 to 96 years $(69.47 \pm 7.45)$. The hospital mortality was associated at the beginning of hospitalization $(p=0.001)$. Correlation was observed between the scores of the GDS-15 and IBAIVD early ( $p=$ $0.008)$ and end of hospitalization ( $p=0.01)$, verifying inverse linear correlation $(p=-0.30)$, but the magnitudes weak ( $p=-0.30$ and $p=-0.28$ respectively). Conclusion: Depressive symptoms were associated with higher mortality, independent of functional capacity. It emphasizes the importance of assessing depressive symptoms in the elderly in hospitals. New studies and prospective analyzes may provide more indicators for the understanding of this research problem.

1 Universidade Federal da Paraíba (UFPB), Centro de Ciências Médicas (CCM), Departamento de Medicina Interna. 2 UFPB, CCM, Curso de Medicina.

Pesquisa realizada no Hospital Universitário Lauro Wanderley (HULW), Universidade Federal da Parába (UFPB). Instituição onde o trabalho foi elaborado: Departamento de Medicina Interna, Centro de Ciências Médicas, Universidade Federal da Paraíba (UFPB), João Pessoa, Paraíba, Brasil.

Endereço para correspondência: Rilva Lopes de Sousa-Muñoz

Departamento de Medicina Interna, Hospital Universitário Lauro Wanderley Campus I, s/n,

Cidade Universitária - 58050-000 - João Pessoa, PB, Brasil

E-mail: rilva@ccm.ufpb.br 


\section{INTRODUÇÃO}

É elevada a prevalência de sintomas depressivos clinicamente significativos em idosos durante uma hospitalização, pois eles apresentam reação de ajustamento à própria internação, à maior gravidade de sua doença e à suposta ameaça de morte. Estima-se que 35\% a $45 \%$ de idosos internados por problemas somáticos apresentam sintomas depressi$\operatorname{vos}^{1-3}$, enquanto pacientes adultos não idosos apresentam esse quadro em $20 \%$ dos casos quando hospitalizados ${ }^{4,5}$. Por outro lado, é de 15\% a prevalência de sintomas depressivos em idosos não hospitalizados ${ }^{6}$.

Sintomas depressivos podem fazer parte de um quadro de depressão evidente (diagnóstico) ou de depressão subsindrômica, que é definida como a presença de sintomas depressivos na ausência de critérios diagnósticos para transtorno depressivo maior ou menor ${ }^{7,8}$.

A realização de estudos epidemiológicos sobre a associação entre sintomas depressivos e mortalidade em idosos é complexå ${ }^{9}$. Além da variável representada pela doença somática subjacente e da capacidade funcional do idoso, que estão, ambas, relacionadas à ocorrência de sintomas depressivos $^{10}$, outros fatores também devem ser controlados, como gênero ${ }^{11}$ e situação socioeconômica ${ }^{12}$. Por outro lado, a incapacidade funcional, por si só, tem sido considerada um fator importante na evolução clínica de idosos hospitalizados ${ }^{13}$.

O problema de pesquisa deste estudo correspondeu à seguinte pergunta: a presença de sintomas depressivos está associada à evolução desfavorável em pacientes idosos hospitalizados, independente da sua situação de capacidade funcional?

O objetivo deste estudo foi avaliar se a presença de sintomas depressivos associa-se à evolução hospitalar desfavorável (aumento da permanência hospitalar e mortalidade antes da alta), independente da sua capacidade funcional, nas enfermarias de clínica médica do Hospital Universitário Lauro Wanderley (HULW), Universidade Federal da Paraíba, João Pessoa, PB.

\section{MÉTODOS}

O estudo seguiu um delineamento de coorte prospectivo. Foi realizado um seguimento dos pacientes idosos internados nas enfermarias de Clínica Médica do HULW para mensuração das variáveis do estudo em dois momentos da evolução hospitalar (segundo dia de internamento e no dia da alta).

Foram considerados idosos os pacientes com idade igual ou superior a 60 anos, de acordo com a Política Nacional de Saúde do Idoso do Ministério da Saúde. O recrutamento foi feito de modo acidental, com seleção continuada dos pacientes idosos consecutivamente internados nas enferma- rias de Clínica Médica do HULW, no período entre 18 de outubro de 2008 a 8 de março de 2009.

Foram adotados os seguintes critérios de inclusão: a) paciente com idade igual ou superior a 60 anos; b) admissão nas enfermarias de clínica médica do HULW para tratamento; e c) consentimento por escrito para participação na pesquisa. Os critérios de exclusão foram: a) qualquer impossibilidade de comunicação por parte do paciente, como afasia, coma, dificuldade de fonação ou deficiência auditiva grave; e b) presença de dor no momento da entrevista.

O tamanho mínimo da amostra foi calculado de acordo com Richardson (1999), tomando-se por base uma estimativa de prevalência de $45 \%$ de transtornos depressivos em pacientes idosos internados por doenças crônicas em enfermarias gerais. O tamanho da amostra foi calculado com base em um universo finito, considerando o número de idosos internados em 2007 nas enfermarias de clínica médica do mesmo serviço onde se realizou a presente pesquisa ${ }^{14}$. A partir de uma amostragem acidental, com nível de confiança $\left(\sigma^{2}\right)$ de 95\% e erro de estimação $\left(E^{2}\right)$ de $4 \%$, chegou-se ao número mínimo necessário de 70 pacientes para a amostra.

Os instrumentos utilizados na coleta de dados foram a Escala de Depressão Geriátrica (EDG), versão breve (15 itens, EDG-15), e o Índice de Barthel para Incapacidade em Atividades da Vida Diária (IBAIVD).

A EDG, na sua versão breve (15 itens), validada no Brasil ${ }^{15}$ e já empregada na mesma população-alvo ${ }^{14}$, foi escolhida por causa de suas características de validade e fidedignidade e pelo fato de poder ser aplicada por pesquisadores sem formação psiquiátrica específica. Embora seja de autoaplicação, nesta pesquisa a EDG-15 foi administrada no formato de formulário, e as questões foram lidas em voz alta, para contornar o problema da baixa escolaridade dos participantes $^{14}$. Foi realizado um pré-teste dos instrumentos, que foram aplicados a 10 pacientes (10\% da amostra).

Adotou-se a classificação de Yesavage et al. para classificação da pontuação global (soma de todos os itens) obtida com a aplicação da versão breve da EDG: entre 0 e 4 pontos, os idosos foram considerados eutímicos (provavelmente não deprimidos); pontuação entre 5 e 10 pontos indicou suspeita de depressão leve; e 11 ou mais pontos foi escore compatível com possível depressão moderada/grave ${ }^{16}$.

O IBAIVD foi adaptado no Brasil para medir o grau de incapacidade funcional ${ }^{17}$. Por meio desse instrumento, são avaliadas 10 atividades básicas da vida diária - alimentação, transferência entre a cama e o sanitário, mobilidade (andar sem auxílio de pessoas e ou de objetos), asseio pessoal, banho, vestuário, ato de subir ou descer escadas e controle dos esfíncteres anal e vesical. O escore total resultante da soma de todos os itens pode variar de 0 a 20 pontos. Os idosos que obtiveram entre 0 e 5 pontos foram considerados como portadores de dependência total; entre 6 e 10 pontos, dependência grave; entre 11 e 15 pontos, dependência moderada; 
entre 16 e 19 pontos, dependência leve; e com 20 pontos, independência em atividades básicas da vida diária ${ }^{17}$.

Após assinatura do consentimento esclarecido pelos pacientes, os dados foram colhidos mediante entrevistas individuais realizadas à beira do leito nas referidas enfermarias. As entrevistas foram realizadas no segundo dia de internamento e posteriormente no dia da alta hospitalar e tiveram duração de aproximadamente 25 minutos nas duas ocasiões.

Na estatística inferencial, para a comparação das categorias, foram usados testes não paramétricos: teste de MannWhitney, para dados ordinais e intervalares não pareados; teste de Wilcoxon, para dados pareados; teste do qui-quadrado ou teste exato de Fisher, para dados categóricos; correlação de Spearman, para associação linear bivariada. Foram realizados cruzamentos simples entre cada variável (gênero, faixas de idade, renda, estado civil e procedência) e sintomas depressivos (como um escore global, numérico) por meio de regressões lineares simples e, depois, uma análise multivariada pela regressão linear múltipla. O nível de significância estatística adotado em todos os testes foi de $5 \%$.

\section{RESULTADOS}

Dos 153 pacientes idosos internados nas enfermarias de Clínica Médica do HULW durante o período de cinco meses de coleta de dados, 53 preencheram critérios de exclusão.

A idade dos pacientes incluídos na pesquisa variou entre 60 e 96 anos $(69,47 \pm 7,45)$, sendo 59\% $(n=59)$ do sexo feminino. Na presente amostra, $17 \%$ ainda mantinham atividades laborativas e $16 \%$ referiram não receber proventos da Previdência Social. As frequências referentes às variáveis demográficas estão apresentadas na tabela 1.

O tempo de permanência hospitalar variou de 4 a 105 dias $(25,6 \pm 16,7)$. No dia da alta, $73 \%$ dos pacientes haviam melhorado, 12\% haviam morrido, $12 \%$ tiveram alta a pedido e 3\% foram transferidos para outros hospitais. A permanência hospitalar média dos pacientes que morreram foi de 22,7 dias $( \pm 12,6)$.

As causas de óbito registradas nos prontuários foram as seguintes: insuficiência cardíaca congestiva $(n=3)$, insuficiência respiratória aguda $(n=2)$, neoplasia pancreática ( $n$ $=1$ ), broncopneumonia $(n=1)$, infarto agudo do miocárdio $(n=1)$, neoplasia pulmonar $(n=1)$, tumor de mediastino ( $n$ $=1$ ), encefalopatia hepática $(n=1)$ e morte súbita de causa não determinada $(n=1)$.

Os sintomas depressivos mais frequentemente encontrados por meio da aplicação da EDG-15 no início da hospitalização estão demonstrados na tabela 2.

Os escores obtidos mediante a aplicação da EDG-15 no segundo dia de hospitalização variaram entre 0 e 11 (4,34 \pm 2,33). Observou-se que $23 \%$ dos pacientes atingiram o ponto de corte estabelecido previamente para a presença de suspeita de depressão (EDG > 5) nessa primeira entrevista. A classificação sintomatológica baseada em Yesavage (1983) resultou nas seguintes frequências: ausência de sintomas depressivos clinicamente significativos (77\%), sintomatologia depressiva leve (21\%) e sintomatologia depressiva moderada/grave (2\%).

Dos 23 pacientes que atingiram o ponto de corte da EDG-15, 16 (69,5\%) foram examinados pelo médico psiquiatra. Nessa avaliação, foram afastados transtornos psiquiátricos em sete (44\%) dos idosos que atingiram os cinco pontos na aplicação da EDG-15. Sete pacientes não foram avaliados porque tiveram alta hospitalar $(n=4)$, óbito $(n=1)$ ou transferência para o centro de terapia intensiva $(n=2)$, antes da realização da consulta psiquiátrica.

Não se observou diferença estatística significativa ( $p=$ NS) nas pontuações medianas da EDG-15 quanto ao gênero, embora os escores médios das mulheres $(4,6 \pm 2,4)$ tenham sido discretamente superiores aos dos homens $(3,9 \pm 2,1)$.

Tabela 1. Variáveis sociodemográficas dos pacientes idosos internados nas enfermarias de Clínica Médica do Hospital Universitário Lauro Wanderley

\begin{tabular}{lc}
\hline Variáveis demográficas & $\mathbf{f} / \%$ \\
\hline Gênero & \\
Masculino & 41 \\
Feminino & 59 \\
Idade & 58 \\
$60-69$ anos & 33 \\
$70-79$ anos & 9 \\
$>80$ anos & \\
Estado civil & \\
Casado & 54 \\
Viúvo & 32 \\
Solteiro & 11 \\
Divorciado & 3 \\
Procedência & \\
Capital & \\
Interior & 58 \\
Renda familiar & 42 \\
<1 salário & \\
2-3 salários & \\
$>3$ salários & 76 \\
\hline
\end{tabular}

Tabela 2. Descrição dos itens mais encontrados por meio da aplicação da EDG-15 no início da internação de pacientes idosos internados nas enfermarias de Clínica Médica do Hospital Universitário Lauro Wanderley

\begin{tabular}{lc}
\hline Itens da EDG-15 & $\begin{array}{c}\text { Frequências } \\
\text { (f/\%) }\end{array}$ \\
\hline EDG-9 = Sente-se desamparado & 65 \\
EDG-12 = Acha ruim o modo como se encontra & 62 \\
EDG-6 = Sente medo de que algo ruim Ihe aconteça & 51 \\
EDG-2 = Na semana antes da internação deixou de fazer & 59 \\
coisas que antes fazia habitualmente & \\
EDG-4 = Sente-se aborrecido & 45 \\
EDG-3 = Sente que a vida está vazia & 32 \\
EDG-10 = Acha que sua memória está pior que a dos outros & 18 \\
\hline
\end{tabular}


Não se observou correlação entre escores da EDG-15 e a idade dos pacientes $(p=N S)$.

No grupo de idosos portadores de sintomatologia depressiva clinicamente importante $(n=23)$, a duração da permanência hospitalar variou de 5 a 68 dias $(28,6 \pm 17,1)$, o que não diferiu significativamente $(p=N S)$ da permanência dos 77 pacientes que não atingiram o ponto de corte na EDG-15 $(24,3 \pm 16,6)$. A permanência hospitalar também não diferiu quando se avaliou nesses dois grupos o número de dias de internação em função da idade e do gênero. A duração da internação também não diferiu quanto à idade $(p=N S)$ e ao gênero ( $p=$ NS) em comparações independentes da presença de sintomas depressivos clinicamente significativos.

Analisando os 75 pacientes nos quais a EDG-15 foi aplicada no início e no final da internação, observou-se diferença estatisticamente significativa $(p=0,004)$ nas pontuações da EDG-15 entre a aplicação feita no início da internação $(4,0 \pm$ $2,1)$ e a do momento da alta $(3,32 \pm 1,5)$, indicando diminuição na intensidade dos sintomas entre esses dois momentos da hospitalização.

A mortalidade hospitalar relacionou-se estatisticamente com os escores da EDG-15 no início da internação $(p=0,001)$. A proporção de pacientes que evoluiu para morte durante a internação foi maior entre os doentes que atingiram o ponto de corte (EDG-15 > 5), sendo classificados como portadores de sintomas depressivos de intensidade leve ou moderada/ grave. Essa associação persistiu ao se controlarem estatisticamente as variáveis faixa de idade (60-69; 70-79; 80 ou mais), escolaridade, gênero, estado civil e procedência.

Os escores obtidos por meio da aplicação do IBAIVD no início da internação hospitalar $(n=100)$ variaram de 3 a 20 $(14,4 \pm 9,4)$. Constatou-se que $25 \%$ dos pacientes apresentavam incapacidade grave para realização de suas atividades instrumentais básicas, 21\% apresentavam independência funcional, enquanto 3\% tinham dependência total.

A duração da permanência hospitalar também não se relacionou com a capacidade funcional avaliada no início da internação ( $p=N S)$. Essa falta de associação persistiu quando se controlaram as variáveis faixas de idade e gênero. Considerando-se apenas os escores dos pacientes que foram submetidos às duas mensurações pelo IBAIVD (início e final da internação) - 75 pacientes -, verificou-se que houve aumento significativo ( $p=0,0001)$ na magnitude dessas pontuações entre a primeira entrevista $(14,9 \pm 5,0)$ e a segunda $(16,7 \pm 3,6)$, denotando melhora da capacidade funcional para realização das atividades instrumentais ao longo da evolução hospitalar.

Os escores do IBAIVD aplicado no início da internação não diferiram significativamente $(p=N S)$ nos pacientes que morreram durante a hospitalização $(n=12 ; 15,1 \pm 5,0)$ em relação aos que sobreviveram ( $n=88 ; 13,5 \pm 4,5)$.

Observou-se correlação estatisticamente significativa entre os escores da EDG-15 e do IBAIVD no início $(p=0,008)$ e no final da internação $(p=0,01)$, verificando-se correlações lineares inversas, porém de magnitudes fracas $(r=-0,30$ e $-0,28$, respectivamente), como pode ser observado nas figuras 1 e 2 .

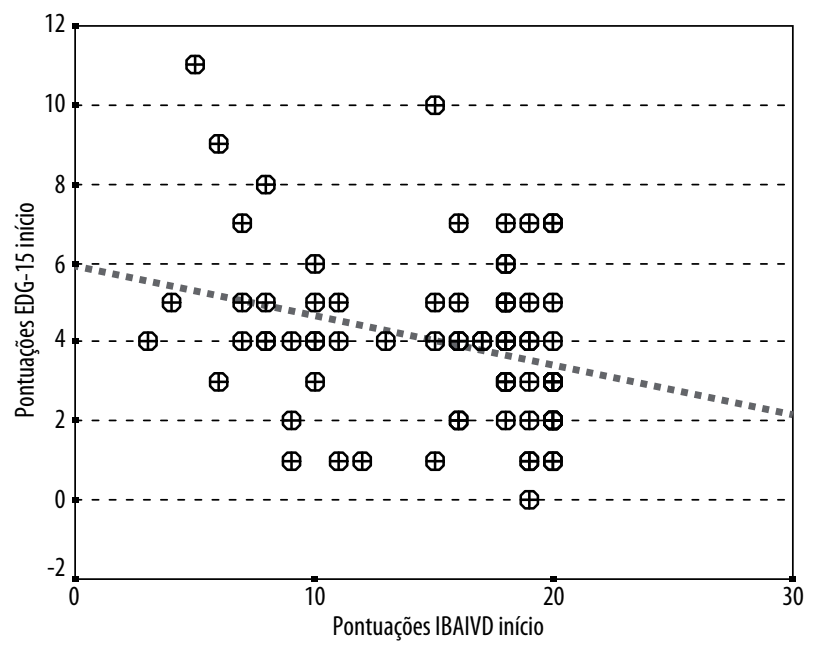

Figura 1. Relação inversa entre a capacidade funcional e sintomatologia depressiva no início da internação $(n=75)$ em pacientes idosos internados nas enfermarias de Clínica Médica do Hospital Universitário Lauro Wanderley.

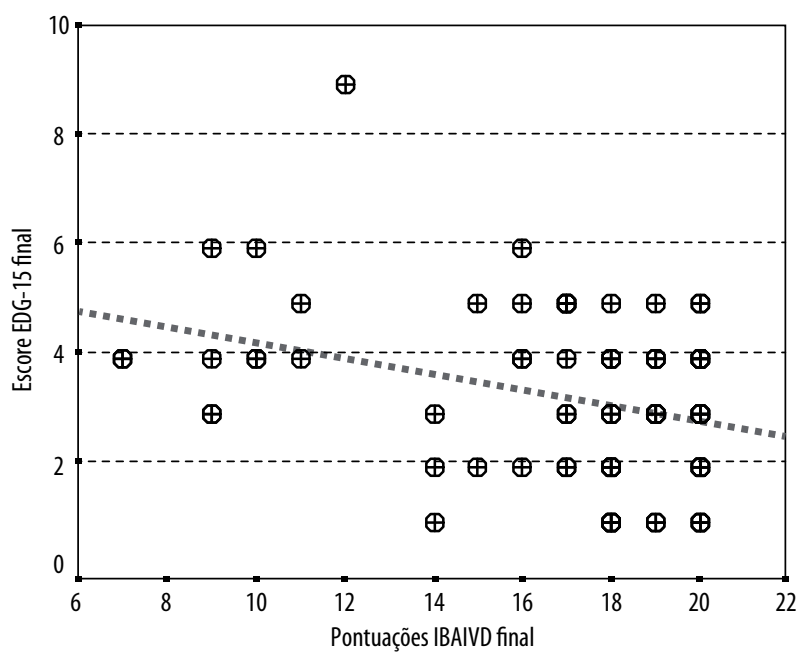

Figura 2. Relação inversa entre a capacidade funcional e sintomatologia depressiva no final da internação $(n=75)$ em pacientes idosos internados nas enfermarias de Clínica Médica do Hospital Universitário Lauro Wanderley.

\section{DISCUSSÃO}

A frequência estimada de sintomatologia depressiva clinicamente significativa observada na presente amostra foi menor que as prevalências reportadas por outros autores no Brasil, que variaram entre 35\% e 45\% em idosos internados em en- 
fermarias gerais para tratamento de doenças crônicas ${ }^{18,19}$. Em outro estudo, encontrou-se prevalência mais elevada, contudo esses autores não empregaram um instrumento padronizado e validado para avaliação de sintomas depressivos ${ }^{20}$. A frequência de incapacidade funcional encontrada também corrobora estudos anteriores envolvendo idosos hospitalizados em enfermarias gerais ${ }^{21,22}$.

A associação observada entre mortalidade hospitalar com a intensidade da sintomatologia depressiva no início da internação é compatível com resultados relatados por outros autores $^{23-25}$. Aceita-se atualmente que depressão e sintomas depressivos são fatores preditivos de maior mortalidade em idosos portadores de doenças crônicas, sobretudo as cardiovasculares ${ }^{26,27}$.

A associação entre sintomatologia depressiva e ocorrência de óbito não teve como variável interveniente a incapacidade funcional. Uma vez que as pontuações referentes aos sintomas depressivos e à incapacidade funcional correlacionaram-se entre si, havia a possibilidade de a primeira variável relacionar-se com mortalidade por depender da última variável (incapacidade funcional), sendo esta o fator mais importante no desfecho. $O$ controle dos escores referentes à incapacidade funcional não modificou a relação encontrada.

A relação entre sintomatologia depressiva e mortalidade também não dependeu das variáveis idade, gênero, etnia, escolaridade e procedência. Dados resultantes de estudos em que são controlados os fatores de risco relacionados à idade, saúde física, recursos materiais e fatores sociais sugerem que o aumento da idade em si não é um fator de risco para depressão $0^{8,17}$.

A ausência de associação de sintomatologia depressiva com permanência hospitalar é discordante do que afirmam outros autores ${ }^{23}$, que consideram que os sintomas depressivos influenciam a duração da permanência hospitalar dos pacientes idosos pela ação desses sintomas sobre a imunidade celular, favorecendo a instalação de infecções, além da possível alteração da função neuroendócrina e da atividade do sistema nervoso autônomo, o que determinaria recuperação mais lenta. No entanto, ainda que a presença de sintomatologia depressiva em idosos influencie a evolução hospitalar dos pacientes após a alta hospitalar, Cullum et al. (2008) também não encontraram associação entre duração da permanência com aquela variável ${ }^{25}$.

A melhora da sintomatologia depressiva entre o início e o final do internamento indica possivelmente o resultado da melhora do estado clínico dos pacientes e a perspectiva percebida da alta hospitalar iminente. Esse achado não foi estudado por outros autores para fins de comparação, mas se trata de um resultado esperado do ponto de vista clínico.

A correlação entre sintomas depressivos e incapacidade funcional no início e no final da internação corrobora estudo anterior ${ }^{28}$, em que se enfatiza a importância clínica dessa associação no doente idoso portador de doença crônica. As correlações de magnitudes fracas encontradas no presente estudo podem refletir a influência de outras variáveis intervenientes sobre essa relação, por exemplo, a gravidade da doença que motivou o internamento. Contudo, idade e gênero não influenciaram essa associação. Nesse sentido, as doenças de base, que não foram um fator controlado neste estudo, podem ter potencializado fortemente a incapacidade funcional nos idosos ${ }^{29}$.

Por outro lado, a incapacidade funcional pode ser causa ou consequência de sintomatologia depressiva ${ }^{30}$. A incapacidade funcional, quando associada a uma doença física grave, pode ser importante fator de risco para depressão. Sabese, todavia, que os sintomas de doenças físicas e depressão são fatores de risco para a incapacidade funcional também, e não é rara a associação das três condições nos idosos ${ }^{13}$.

A duração da permanência hospitalar também não se relacionou com a capacidade funcional avaliada no início da internação, o que não corrobora os achados de Conde-Martel et al., que encontraram tal associação ${ }^{31}$. Por outro lado, em outro estudo, constatou-se elevada mortalidade seis meses após a internação hospitalar em pesquisa realizada com pacientes idosos que apresentavam incapacidade funcional importante durante a internação ${ }^{29}$.

No presente trabalho, já no final da hospitalização, foi possível observar melhora significativa da capacidade funcional, em relação ao início do internamento, de forma semelhante ao que ocorreu com a sintomatologia depressiva. Essas duas variáveis se correlacionaram significativamente nos dois momentos da mensuração ao longo do internamento.

\section{CONCLUSÃO}

Sintomatologia depressiva associou-se a maior mortalidade, independente da capacidade funcional, faixa de idade, gênero, estado civil, escolaridade e procedência. Foi constatada melhora significativa da sintomatologia depressiva e da incapacidade funcional entre o início e o final da hospitalização. Ressalta-se a importância da avaliação de sintomatologia depressiva nos idosos no contexto hospitalar. Novos estudos e análises prospectivas com maior número de medidas e controle de variáveis intervenientes poderão oferecer mais indicadores para o entendimento desse problema.

\section{CONTRIBUIÇÃO INDIVIDUAL}

Rilva Lopes de Sousa-Muñoz - Trabalhou na concepção, planejamento, análise e interpretação dos dados, na redação do artigo e em sua revisão crítica.

Ezemir Dantas Fernandes Junior, Divany de Brito Nascimento e Bruno Braz Garcia - Trabalharam na análise e interpretação dos dados e na redação do artigo. 
Iramirton Figuêredo Moreira - Trabalhou na concepção, planejamento, coleta de dados e revisão intelectual do artigo.

\section{CONFLITOS DE INTERESSE}

Não há conflitos de interesse

\section{AGRADECIMENTOS}

Agradecemos ao Programa de Bolsas de Iniciação Científica $\mathrm{PIBIC/CNPq/UFPB}$.

\section{REFERÊNCIAS}

1. Dennis M, Kadri A, Coffey J. Depression in older people in the general hospital: a systematic review of screening instruments. Age Aging. 2012;41(2):148-54.

2. Siqueira GR, Vasconcelos DT, Duarte GC, Arruda IC, Costa JAS, Cardoso RO. Análise da sintomatologia depressiva nos moradores do Abrigo Cristo Redentor através da aplicação da Escala de Depressão Geriátrica (EDG). Ciênc Saúde Coletiva. 2009;14(1):253-9.

3. Hoffmann EJ, Ribeiro F, Farnese JM. Sintomas depressivos e fatores associados entre idosos residentes em uma comunidade no norte de Minas Gerais, Brasil. J Bras Psiquiatr. 2010;59(3):190-7.

4. Austin P, Wiley S, McEvoy PM, Archer L. Depression and anxiety in palliative care inpatients compared with those receiving palliative care at home. Palliat Support Care. 2011;9(4):393-400.

5. Medeiros PCS, Gioia-Martins DM, Hamzeh SA. Avaliação psicológica de depressão em pacientes internados em enfermaria de hospital geral. Psicologia: Teoria e Prática. 2009:11(1):128-41.

6. Pinho MX, Custodio 0, Makdisse M. Incidência de depressão e fatores associados em idosos residentes na comunidade: revisão de literatura. Rev Bras Geriatr Gerontol. 2009;12 (1):123-40.

7. Pibernik-Okanovic M, Ajdukovic D, Lovrencic MV, Hermanns N. Does treatment of subsyndromal depression improve depression and diabetes related outcomes: protocol for a randomized controlled comparison of psycho-education, physical exercise and treatment as usual. Trials. 2011;12:17.

8. Lyness JM, Kim J, Tang W, Tu X, Conwell Y, King DA, et al. The clinical significance of subsyndromal depression in older primary care patients. Am J Geriatr Psychiatry. 2007;15(3):214-23

9. Vest MT, Murphy TE, Araujo KL, Pisani MA. Disability in activities of daily living, depression, and quality of life among older medical ICU survivors: a prospective cohort study. Health Qual Life Outcomes. 2011;9(9):2-10.

10. Mezuk B, Lohman M, Dumenci L, Lapane KL. Are depression and frailty overlapping syndromes in mid- and late-life? A latent variable analysis. Am J Geriatr Psychiatry. 2012;20(6):457-64.

11. Eastwood JA, Moser DK, Riegel BJ, Albert NM, Pressler S, Chung ML, et al. Commonalities and differences in correlates of depressive symptoms in men and women with heart failure. Eur J Cardiovasc Nurs. 2012;6(2):146-52.
12. Worth P, Olesen SC, Leach LS. The role of hardship in the association between socio-economic position and depression. Aust N Z J Psychiatry. 2012;46(4):364-73.

13. Olafiranye 0 , Jean-Louis G, Antwi M, Zizi F, Schaw R, Brimah P. Functional capacity is a better predictor of coronary heart disease than depression or abnormal sleep duration in black and white Americans. Sleep Med. 2012;13(6):728-31.

14. Sousa-Muñoz, RL, Medeiros JGM, Moura ACL, Souza CLM, Moreira IF. Validade e fidedignidade da Escala de Depressão Geriátrica na identificação de idosos deprimidos em um hospital geral. J Bras Psiquiatr. 2007;56(2):102-7.

15. Stoppe Jr AJR, Jacob WF, Louzã-Neto MRN. Avaliação de depressão em idosos através da "Escala de Depressão em Geriatria": resultados preliminares. Rev ABP-APAL. 1994;16:149-53.

16. Yesavage JA, Brink TL, Rose TL, Lum 0, Huang V, Leirer V0. Development and validation of a geriatric screening scale. J Psychiatr Res. 1983;17(8):37-49.

17. Minosso, JSM, Amendola F, Alvarenga MRM, Oliveira MAC. Validação, no Brasil, do Índice de Barthel em idosos atendidos em ambulatórios. Acta Paul Enferm. 2010;23(2):218-23.

18. Ferarri JF, Dalacorte RR. Uso da Escala de Depressão Geriátrica de Yesavage para avaliar a prevalência de depressão em idosos hospitalizados. Sci Med. 2007;17(1):3-8.

19. Porcu M, Scatamburlo VM, Albrecht NR, Silva SP, Vallim FL, Araújo CL, et al. Estudo comparativo sobre a prevalência de sintomas depressivos em idosos hospitalizados, institucionalizados e residentes na comunidade. Acta Sci. 2008;24(3):712-3.

20. Gazalle FK, Lima MS, Tavares BF, Hallal PC. Sintomas depressivos e fatores associados em população idosa no Sul do Brasil. Rev Saude Publica. 2004;38(3):365-71.

21. Murray ET, Hardy R, Strand BH, Cooper R, Guaralnik JM, Kuh D. Gender and life course OC cupational social class differences in trajectories of functional limitations in midlife: findings from the 1946 British birth cohort. J Gerontol A Biol Sci Med Sci. 2011;66(12):1350-9.

22. Abdulraheem IS, Oladipo AR, Amodu MO. Prevalence and correlates of physical disability and functional limitation among elderly rural population in Nigeria. J Aging Res. 2001;36(9):2-24

23. Prina AM, Deeg D, Brayne C, Beekman A, Huisman M. The association between depressive symptoms and non-psychiatric hospitalisation in older adults. PLoS One. 2012;7(4):e34821.

24. Sánchez E, Vidán MT, Serra JA, Fernandés-Avilléz, F, Bueno H. Prevalence of geriatric syndromes and impact on clinical and functional outcomes in older patients with acute cardiac diseases. Heart. 2011;97(19):1602-6

25. Cullum S, Metcalfe C, Todd C, Brayne, C. Does depression predict adverse outcomes for older medical inpatients? A prospective cohort study of individuals screened for a trial. Age Ageing. 2008;37(6):690-5

26. Kamphuis MH, Geerlings MI, Giampaoli S. The association of depression with cardiovascular mortality is partly explained by health status. The FINE Study. J Affect Disord. 2009;114(1-3):184-92.

27. Kamphuis MH, Kalmijn S, Tijhuis MA. Depressive symptoms as risk factor of cardiovascular mortality in older European men: the Finland, Italy and Netherlands Elderly (FINE) study. Eur J Cardiovasc Prev Rehabil. 2006:13(2):199-206.

28. Vivan AS, Argimon IIL. Estratégias de enfrentamento, dificuldades funcionais e fatores associados em idosos institucionalizados. Cad Saude Publica. 2009;25(2):436-44.

29. Leão SSC, Lopes AA, Souza AAM, Dalan SAO, Lourenção Jr A, Stolg NAG. Preditores de mortalidade em pacientes idosos após seis meses de alta hospitalar. Rev Med PUCRS. 2002;12(1):12-6

30. Lima MTR, Silva RS, Ramos LR. Fatores associados à sintomatologia depressiva numa coorte urbana de idosos. J Bras Psiquiatr. 2009;58(1):1-7.

31. Conde-Martel A, Hemmersbach-Miller M, Anía Lafuente BJ, Sujanani Afonso N, SerranoFuentes M. Prevalence of depressive symptoms in hospitalized elderly medical patients. Rev Esp Geriatr Gerontol. 2013;S0211-139X(12)00231-4. 